\title{
CARACTERÍSTICAS CLÍNICO-EPIDEMIOLÓGICAS Y PATRONES DE PRESCRIPCIÓN PARA QUEMADURAS EN TRES HOSPITALES DE LIMA, PERÚ
}

\author{
Guillermo Martin Wiegering Cecchi ${ }^{1, a}$, Enrique Rios Hidalgo ${ }^{2, b}$, José Víctor Córdova Orrillo ${ }^{3, b}$, \\ Juan Rafael Ludeña Muñoz ${ }^{1, b}$, Cecilia A. Medina ${ }^{4, c}$
}

\begin{abstract}
RESUMEN
Con el objetivo de describir las características clínico-epidemiológicas y los patrones de prescripción médica de pacientes con quemaduras de primer y segundo grado que acudieron a tres hospitales de referencia de Lima, se realizó un estudio transversal donde se recogieron datos demográficos, antecedentes médicos, evaluación clínica y tratamiento recibido en 561 participantes. El uso de antibióticos y de agentes humectantes se dio en $64,7 \%$ y $4,2 \%$ en los centros de atención inmediata; y en $41,7 \%$ y $44,7 \%$ en los servicios de atención especializada en quemaduras. La sulfadiazina argéntica fue el antibiótico tópico más utilizado en los servicios de atención inmediata, en comparación con los servicios de quemados $(80,2 \%$ vs $34,5 \%)$. El manejo de quemaduras fue más exhaustivo en los servicios de quemados que en los de atención inmediata. Asimismo, más de un cuarto de los pacientes que acudieron por emergencia lo hicieron luego de 24 horas de ocurrida la quemadura.
\end{abstract}

Palabras clave: Quemaduras; Unidades de Quemados; Tratamiento; Perú (fuente: DeCS BIREME)

\section{CLINICAL-EPIDEMIOLOGICAL CHARACTERISTICS AND PRESCRIBING PATTERNS FOR BURNS IN THREE HOSPITALS IN LIMA, PERU}

\begin{abstract}
In order to describe the clinical-epidemiological characteristics and medical prescription patterns of patients with first- and second-degree burns who visited three reference hospitals in Lima, a cross-sectional study was carried out to collect data on demographics, medical history, clinical evaluation, and treatment received by 561 participants. The use of antibiotics and moisturizing agents was $64.7 \%$ and $4.2 \%$ in immediate care centers; and $41.7 \%$ and $44.7 \%$ in specialized burn-care services. Argenic sulfadiazine was the most commonly used topical antibiotic in immediate care services compared to burned units $(80.2 \%$ vs. $34.5 \%)$. Burn management was more comprehensive in burn services than in immediate care. Also, more than a quarter of the patients who sought emergency care did so within 24 hours of the burn.
\end{abstract}

Keywords: Burns; Burn Units; Treatment; Peru (source: MeSH NLM).

\section{INTRODUCCIÓN}

Las lesiones causadas por quemaduras constituyen un problema de salud a nivel global que afecta a todos los grupos etarios, no sólo por la frecuencia en que ocurren, sino que de acuerdo con su severidad pueden ser incapacitantes y tener una alta mortalidad, y a la vez, generar un impacto económico desfavorable para el país.

En Perú las estadísticas no son precisas, ya que los estudios relacionados a esta patología se han dirigido principalmente a la población infantil ${ }^{(1,2)}$, grandes quemados ${ }^{(3)}$ y quemaduras

\footnotetext{
Hospital Nacional Arzobispo Loayza. Lima, Perú.

Hospital Nacional Daniel Alcides Carrión. Lima, Perú.

Complejo Hospitalario San Pablo. Lima, Perú.

Sanofi Aventis del Perú. Lima, Perú.

a Médico cirujano, especialista en Cirugía plástica y Reparadora, doctor en Medicina; ${ }^{b}$ médico cirujano, especialista en Cirugía plástica y Reparadora; ${ }^{c}$ médico cirujano, especialista en Endocrinología

Recibido: 05/05/2018 Aprobado: 20/02/2019 En línea: 13/03/2019
}

Citar como: Wiegering Cecchi GM, Rios Hidalgo E, Córdova Orrillo JV, Ludeña Muñoz JR, Medina CA. Características clínico-epidemiológicas y patrones de prescripción para quemaduras en tres hospitales de Lima, Perú. Rev Peru Med Exp Salud Publica. 2019;36(1):68-73.doi:10.17843/rpmesp.2019.361.3649. 
solares (4); sin embargo, según Global Health Data Exchange ${ }^{(5)}$, se estima que la incidencia de quemaduras menores del $20 \%$ de la superficie corporal sin compromiso de las vías áreas para el 2017 fue de 113 casos nuevos por cada 100000 habitantes, siendo el fuego, el calor y las sustancias calientes los principales agentes causales.

En Perú, hay escasa información sobre el uso de antibióticos tópicos en quemaduras. Sin embargo, las agencias reguladoras están apoyando la realización de estudios cada vez más complejos con la finalidad de mejorar la eficacia y seguridad de los medicamentos usados frecuentemente ${ }^{(6)}$.

En este contexto, es apropiado generar evidencia epidemiológica sobre los patrones de uso de medicamentos en los pacientes con quemaduras, incluyendo el uso de antibióticos tópicos. El presente estudio pretende describir las características clínico-epidemiológicas y los patrones de prescripción médica en pacientes adultos con quemaduras de primer $y$ de segundo grado atendidos en tres hospitales de referencia en Lima, Perú.

\section{EL ESTUDIO}

Se realizó un estudio transversal descriptivo, entre marzo del 2014 y abril del 2016 para evaluar las características clínicoepidemiológicas y los patrones de prescripción médica en pacientes adultos con quemaduras de primer y/o segundo grado en dos hospitales públicos de tercer nivel: el Hospital Nacional Arzobispo Loayza y el Hospital Nacional Daniel Alcides Carrión, y una clínica privada: Clínica San Pablo en Lima, Perú. Todos los centros hospitalarios contaban con servicios de emergencia y servicios especializado en quemaduras.

Los criterios de inclusión fueron: pacientes de ambos sexos con edades entre 18 y 80 años, con diagnóstico inicial de quemaduras de primer grado, segundo grado o ambos, atendidos en centros de atención inmediata (incluye el servicio de emergencia del mismo hospital u otros hospitales, clínicas, postas de salud, consultorios particulares, farmacias, casa (automedicación), etc.) y en los servicios especializados en quemados de los centros hospitalarios participantes en el estudio. Fueron excluidos: pacientes que no firmaron el consentimiento informado.

Una vez que el participante firmó el consentimiento informado, el médico del estudio recogió la información, la cual provenía mayormente de la historia clínica; sin embargo, algunos datos fueron corroborados en la entrevista que el paciente tuvo con los investigadores. Esto se dio para las siguientes variables: nivel de educación, procedencia, ocupación, lugar donde ocurrió la quemadura y lugar de atención inicial. Posteriormente, la información fue ingresada a formularios electrónicos.

\section{MENSAJES CLAVE}

Motivación para realizar el estudio. Falta de estudios que proporcionen información estadística sobre las características clínico-epidemiológicas y los patrones de prescripción en pacientes quemados de Perú.

Principales hallazgos. Las características clínico-epidemiológicas de los pacientes con quemaduras fueron similares a la de otros países de Latinoamérica. El manejo de las quemaduras fue más exhaustivo en los servicios de quemados, donde se dio más importancia a la reepitelizacion y al manejo antinflamatorio, y menos al tratamiento con antibióticos tópicos.

Implicancias. Es necesario implementar estrategias para transmitir el manejo adecuado de las quemaduras al personal de los servicios de emergencia.

Se realizó el cálculo de frecuencias y porcentajes para las variables discretas, y de la media/mediana con la desviación estándar (DE) / rango intercuartil (RIC) para las variables continuas. Dado que no hay estudios a nivel nacional que muestren estadísticas sobre el número de quemados o que brinden información sobre el manejo de las quemaduras en instituciones que cuenten con una unidad de quemados, se decidió enrolar a todos los pacientes de las tres sedes hospitalarias que cumplían con los criterios de inclusión durante 25 meses, periodo para el cual se contó con financiamiento.

El protocolo de estudio obtuvo la aprobación de los comités de ética de cada centro hospitalario. La información se recolectó de manera confidencial, sin exponer información personal de los participantes.

\section{HALLAZGOS}

Se reclutó un total de 577 participantes, de los cuales uno no brindó su consentimiento informado, y en 15 no se pudo obtener características de la quemadura en la historia clínica, por lo que finalmente el análisis se realizó en 561 participantes.

La mayoría fueron mujeres $(57,8 \%, 324)$, con una edad media de 38,7 años. El $77,0 \%(432)$ tenía educación secundaria completa o superior, el $94,5 \%$ (530) reportó que vivía en la ciudad de Lima, y el 79,7\% (447) no presentaban antecedentes de enfermedades previas. El 77,9\% (437) fueron atendidos en el Hospital Nacional Arzobispo Loayza (Tabla 1).

El promedio del tiempo transcurrido entre la hora en que el paciente fue atendido en el servicio de atención primaria y el cuidado recibido en el servicio especializado fue de 26 horas (RIC: 3-96 horas). El 53,8\% (302) de los pacientes acudieron primero a la sala de emergencias del hospital, de este grupo el $26,2 \%$ (79) acudieron después de 24 horas 
Tabla 1. Características demográficas de pacientes quemados que se atendieron en tres centros hospitalarios de Lima

\begin{tabular}{lc}
\hline Características & $\mathbf{n}(\%)$ \\
\hline Edad (años) & $38,7(17,6)$ \\
Sexo & \\
$\quad$ Masculino & $237(42,2)$ \\
\hline Femenino & $324(57,8)$ \\
\hline Nivel de educación & \\
$\quad$ Secundaria incompleta o menos & $127(22,6)$ \\
\hline Secundaria completa & $229(40,8)$ \\
\hline Superior & $203(36,2)$ \\
\hline No hay información & $2(0,4)$ \\
\hline Procedencia & \\
\hline Lima & $530(94,5)$ \\
\hline Provincia & $31(5,5)$ \\
\hline Ocupación & \\
\hline Estudiante & $76(13,5)$ \\
\hline Ama de casa & $177(31,6)$ \\
\hline Trabajo de oficina & $76(13,5)$ \\
\hline Otro & $232(41,4)$ \\
\hline Condición médica previa & \\
\hline Saludable & $447(79,7)$ \\
\hline Presenta alguna comorbilidad & $102(18,2)$ \\
\hline No hay información & $12(2,1)$ \\
\hline Sede hospitalaria & \\
\hline Hospital Nacional Arzobispo Loayza & $437(77,9)$ \\
\hline Hospital Nacional Daniel Alcides Carrión & $69(12,3)$ \\
\hline Clínica San Pablo & $55(9,8)$ \\
\hline Media (desviación estándar) & \\
\hline & \\
\hline & \\
\hline
\end{tabular}

de ocurrida la quemadura. El 14,4\% (81) de los pacientes fueron hospitalizados.

La mayoría sufrió lesión por quemadura en el hogar (61,3\%, 344). El contacto con los líquidos calientes fue la causa más común de quemaduras $(50,6 \%, 284)$. La cara $(28,7 \%, 161)$, el antebrazo $(26,6 \%, 149)$ y las manos $(22,6 \%, 127)$ fueron las áreas más frecuentemente afectadas (Tabla 2).

Con respecto a las diferencias entre los centros públicos y privados se encontró un mayor porcentaje de afectación por quemadura en los centros públicos (media de \% de afectación corporal $=5,6 \%$ ) en comparación con el centro privado (media de $\%$ de afectación corporal= 3,4\%), una mayor proporción de los pacientes $(64,9 \%, 326)$ que acudieron a los centros públicos sufrieron quemaduras en su casa, mientras que en el centro privado esto sucedió más en el trabajo $(56.0 \%, 28)$. Por último, el ingreso por emergencia fue más frecuente en el centro privado $(96.0 \%, 48)$, mientras que en los centros públicos la proporción de ingreso por emergencia fue menor $(50,8 \%, 254)$.

Con respecto al tratamiento antibiótico, este fue más frecuente en la atención inmediata con $64,7 \%$ (363), mientras que en el servicio especializado en quemados fue del $41,7 \%$ (234); sin embargo, la forma de administración del tratamiento entre
Tabla 2. Lugares de atención y características de la lesión por quemadura en pacientes que se atendieron en tres centros hospitalarios de Lima

\begin{tabular}{|c|c|}
\hline Características & n (\%) \\
\hline \multicolumn{2}{|l|}{ Lugar de atención } \\
\hline Emergencia & $302(53,8)$ \\
\hline \multicolumn{2}{|l|}{ Tiempo de atención* } \\
\hline 24 horas o antes & $223(73,8)$ \\
\hline Después de 24 horas & $79(26,2)$ \\
\hline \multicolumn{2}{|l|}{ Lugar donde ocurrió la quemadura } \\
\hline Casa & $344(61,3)$ \\
\hline Trabajo & $179(31,9)$ \\
\hline Otro & $29(5,2)$ \\
\hline No hay información & $9(1,6)$ \\
\hline Hospitalizados & $81(14,4)$ \\
\hline \multicolumn{2}{|l|}{ Agente que causo la quemadura } \\
\hline Líquido caliente & $284(50,6)$ \\
\hline Fuego & $196(34,9)$ \\
\hline Superficie caliente & $44(7,9)$ \\
\hline Sustancia química & $12(2,1)$ \\
\hline Electricidad & $6(1,1)$ \\
\hline Otro & $13(2,3)$ \\
\hline No hay información & $6(1,1)$ \\
\hline \multicolumn{2}{|l|}{ Clasificación de la quemadura } \\
\hline Primer grado & $2(0,4)$ \\
\hline Segundo grado & $222(39,6)$ \\
\hline Primer y segundo grado & $324(57,7)$ \\
\hline No hay información & $13(2,3)$ \\
\hline \multicolumn{2}{|l|}{ 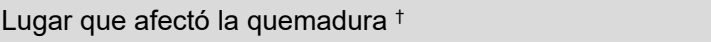 } \\
\hline Cara & $161(28,7)$ \\
\hline Antebrazo & $149(26,6)$ \\
\hline Mano & $127(22,6)$ \\
\hline Tórax & $99(17,7)$ \\
\hline Muslo & $90(16,0)$ \\
\hline Pie & $74(13,9)$ \\
\hline Pantorrilla & $71(12,7)$ \\
\hline Cuello & $46(8,2)$ \\
\hline Brazo & $32(5,7)$ \\
\hline Cabeza & $5(0,9)$ \\
\hline Perineo & $5(0,9)$ \\
\hline Otro & $59(10,5)$ \\
\hline $\begin{array}{l}\text { Porcentaje del cuerpo que afectó la } \\
\text { quemadura } \ddagger\end{array}$ & $4(2-8)$ \\
\hline
\end{tabular}

* De un total de 302 pacientes que acudieron por emergencia

+ Puede incluir más de un lugar de afección

‡ Mediana (rango intercuartil)

la atención inmediata y el servicio especializado fue similar, siendo la vía tópica la más frecuente en ambos casos con una proporción de 91,7\% (333) en los centros de atención inmediata y $88,0 \%$ (206) para la atención especializada. En los servicios de atención inmediata se dio un menor uso de 
los humectantes de piel, siendo del 4,2\% (23); mientras que en los servicios especializados fue del $44,7 \%$ (246) (Tabla 3).

Tanto en los centros de atención inmediata como en los centros de atención especializada, las sulfadiazina argéntica fue el antibiótico tópico más utilizado, aunque con una proporción mayor en la atención inmediata. Asimismo, los fármacos derivados de la penicilina y las cefalosporinas fueron los antibióticos no tópicos más utilizados en la atención inmediata. Mientras que hubo un número similar de prescripciones para derivados de la penicilina, cefalosporinas y quinolonas en los centros de atención especializada (Tabla 4).

En los servicios de atención inmediata, los opiáceos y los medicamentos derivados del ácido propionico fueron los analgésicos usados con mayor frecuencia, mientras que, en los servicios especializados, los opiáceos y los derivados anílicos fueron los medicamentos con mayor prescripción (Tabla 4).

Tabla 3. Lugar de atención y tratamiento de la quemadura en pacientes que se atendieron en tres centros hospitalarios de Lima

\begin{tabular}{lc}
\hline Características & $\mathbf{n}(\%)$ \\
\hline Lugar de atención inmediata & \\
\hline Emergencia del hospital de estudio & $288(51,3)$ \\
\hline Otra clínica u hospital & $96(17,1)$ \\
\hline Posta de salud & $63(11,2)$ \\
\hline Consultorio particular & $11(2,0)$ \\
\hline Casa & $7(1,2)$ \\
\hline Farmacia & $15(2,7)$ \\
\hline Otro & $47(8,4)$ \\
\hline No reportado & $34(6,1)$ \\
\hline Atención inmediata* & \\
\hline Uso de agentes humectantes & $23(4,2)$ \\
\hline Uso de analgésicos & $170(30,3)$ \\
\hline Tratamiento antibiótico (atb) & $363(64,7)$ \\
\hline Tipo de administración atb ${ }^{\dagger}$ & \\
\hline Tópico & $333(91,7)$ \\
\hline Oral & $100(27,5)$ \\
\hline IV/IM & $16(4,4)$ \\
\hline Servicio especializado en quemados* & \\
\hline Uso de agentes humectantes & $246(44,7)$ \\
\hline Uso de analgésicos & $187(33,3)$ \\
\hline Tratamiento antibiótico(atb) & $234(41,7)$ \\
\hline Tipo de administración atb ${ }^{\dagger}$ & $206(88,0)$ \\
\hline Tópico & $46(19,7)$ \\
\hline Oral & $12(5,1)$ \\
\hline IV/IM
\end{tabular}

IV: Intravenoso, IM: Intramuscular

* No se recolectaron todos los tipos de tratamientos, solo los 3 grupos presentados

† Porcentaje del total de pacientes que recibieron tratamiento antibiótico, los pacientes pudieron usar más de un tipo tratamiento antibiótico
Tabla 4. Descripción del tratamiento de acuerdo con el lugar de atención en pacientes que se atendieron en tres centros hospitalarios de Lima

\begin{tabular}{|ccc}
\hline Grupo farmacológico & $\begin{array}{c}\text { Atención } \\
\text { inmediata }\end{array}$ & $\begin{array}{c}\text { Servicio } \\
\text { especializado }\end{array}$ \\
\hline Antibiótico tópico* & $\mathrm{n}=333(\%)$ & $\mathrm{n}=206(\%)$ \\
\hline Derivados de Nitrofuranos & $26(7,8)$ & $36(17,5)$ \\
\hline Mupirocina & $1(0,3)$ & $5(2,4)$ \\
\hline Quinolonas & $4(1,2)$ & $0(0,0)$ \\
\hline Rifamicinas & $16(4,8)$ & $62(30,1)$ \\
\hline Tetraciclinas & $19(5,7)$ & $32(15,5)$ \\
\hline Sulfadiazina argéntica & $267(80,2)$ & $71(34,5)$ \\
\hline Antibiótico no tópico* & $\mathrm{n}=120(\%)$ & $\mathrm{n}=75(\%)$ \\
\hline Aminoglicosidos & $4(3,3)$ & $1(1,3)$ \\
\hline Cefalosporinas & $39(32,5)$ & $24(32,0)$ \\
\hline Lincosamidas & $11(9,2)$ & $4(5,3)$ \\
\hline Penicillinas & $53(44,2)$ & $23(30,7)$ \\
\hline Quinolonas & $13(10,8)$ & $23(30,7)$ \\
\hline Tratamiento analgésico* & $\mathrm{n}=170(\%)$ & $\mathrm{n}=187(\%)$ \\
\hline Derivado anílico & $25(14,7)$ & $50(26,7)$ \\
\hline Ácido propiónico & $43(25,3)$ & $42(22,5)$ \\
\hline Anestésico & $4(2,4)$ & $1(0,5)$ \\
\hline Ácido arilacético & $31(4,7)$ & $6(3,2)$ \\
\hline Coxibs & $8(4,7)$ & $8(4,3)$ \\
\hline Opiáceos & $57(33,5)$ & $77(41,2)$ \\
\hline Oxicam & $2(1,2)$ & $3(1,6)$ \\
\hline
\end{tabular}

* Los pacientes recibieron más de un tipo de tratamiento

\section{DISCUSIÓN}

Nuestro estudio muestra diferencias en el manejo de las quemaduras entre los centros de atención inmediata y los servicios especializados en quemados. Los servicios de atención inmediata no proporcionaron el tratamiento que corresponde al grado y tipo de lesión de la quemadura, utilizando más antibióticos tópicos, y menos uso de agentes humectantes. Esto no ocurre en los servicios especializados en quemados donde se busca una rápida reepitelización, además de evitar la progresión de la lesión, esto generalmente se da en un medio húmedo y libre de infección ${ }^{(7)}$.

Diversas áreas de la superficie corporal estuvieron involucradas en las lesiones por quemaduras, con mayor frecuencia en la cara, los antebrazos y las manos. Esta distribución es consistente con lo reportado en otros estudios ${ }^{(8,9)}$. De la misma manera, el lugar donde mayormente ocurrieron las quemaduras fue en la casa, y el agente predominante fue el líquido caliente.

Lamentablemente, muchas de las quemaduras son atendidas de manera tardía en las salas de emergencias ${ }^{(10)}$. En este estudio, aproximadamente un cuarto de los pacientes 
que acudieron por emergencia se presentaron después de 24 horas de ocurrida la quemadura. Esta presentación tardía podría atribuirse al autotratamiento en el hogar, al uso de medicina tradicional, o a la consulta con farmacéuticos locales; igualmente, los costos de transporte podrían influir en esta decisión

Las lesiones por fuego directo y líquido caliente, continúan siendo las principales causas de quemaduras en el mundo ${ }^{(11,12)}$. En el presente estudio, este patrón persiste, siendo las lesiones de quemaduras causadas por contacto con líquidos calientes y por fuego las causas más comunes. La mayoría de estas lesiones se debieron a accidentes en la casa, esto concuerda con otros estudios realizados en países en desarrollo ${ }^{(13-15)}$. Nuestro estudio encontró que los pacientes atendidos en el centro privado tenían proporciones similares de quemaduras ocurridas en casa y en el trabajo, esto probablemente debido a condiciones más seguras en sus hogares en comparación con los pacientes que se atienden en centros públicos. En cuanto al tratamiento antibiótico, la medicación tópica con sulfonamidas se usó con mayor frecuencia tanto en los servicios de emergencia como en los servicios especializados en quemados. Según lo reportado en la literatura, la sulfadiazina de plata ha sido el antimicrobiano tópico estándar para las quemaduras superficiales ${ }^{(16,17)}$.

Los antibióticos orales, como los medicamentos derivados de la penicilina y los derivados de las cefalosporinas fueron usados en la atención inmediata y en los servicios especializados. Un estudio realizado en Paraguay evaluó el tratamiento antibiótico sistémico en pacientes hospitalizados durante más de 24 horas en los servicios de atención especializada de quemaduras, encontrando que los antibióticos más utilizados fueron la ceftazimida, vancomicina, amikacina y ciprofloxacino, en este mismo estudio se aislaron 79 microorganismos, siendo la Pseudomona aeruginosa la de mayor frecuencia, seguida por Acinetobacter y Klebsiella $s p$. ${ }^{(18)}$. Estos resultados concuerda con nuestro estudio, donde en los servicios de quemados fue utilizado una mayor cantidad de antibióticos de amplio espectro. Un siguiente paso a nuestro estudio, sería realizar un análisis microbiológico para conocer no sólo las cepas de bacterias más frecuentes, sino también sus patrones de resistencia, para así poder brindar una terapia más adecuada.

En los centros de atención inmediata y en los servicios especializados en quemados los tratamientos en el manejo de heridas y el uso de analgésicos fueron similares. Aunque la atención especializada se orientó más a la curación de las heridas con el uso de humectantes que favorecen la reepitelización y con el manejo de fármacos antiinflamatorios. Esta tendencia en el manejo de la atención fue similar a la de otros estudios ${ }^{(19)}$. Entre los medicamentos mayormente usados para el manejo del dolor en quemaduras, se encuentran los opiáceos, que presentan una variedad de opciones disponibles respecto a la potencia, la vía de administración y la duración de la acción, adaptada a cada paciente ${ }^{(20)}$.

Como limitaciones podemos mencionar que, si bien, se han incluido tres centros de atención de pacientes con quemaduras de la ciudad de Lima, los hallazgos no pueden ser extrapolados a otros centros hospitalarios $u$ otras regiones del Perú.

El manejo de las quemaduras fue más exhaustivo en los servicios de atención especializada, donde se dio más importancia al manejo antinflamatorio y menos al tratamiento con antibióticos tópicos. El mejor manejo de los pacientes ocurrió en los centros especializados para pacientes quemados, ya que cuentan con personal con experiencia en el manejo de quemaduras, además de protocolos estandarizados. Es necesario transmitir el conocimiento sobre el manejo de quemaduras al personal de los servicios de emergencia, pese a la alta rotación que tienen estos servicios. Esta información descriptiva representa el estado general del manejo de lesiones por quemaduras en los hospitales de referencia más grandes de Lima, Perú.

En conclusión, el uso de antibióticos tópicos para el tratamiento de las quemaduras fue mayor en los centros de atención inmediata que en los servicios especializados, lo contrario ocurrió con el uso de agentes humectantes. La sulfadiazina argentica fue el antibiótico tópico más utilizado, aunque en menor proporción en los servicios especializados. Aproximadamente, más de un cuarto de los pacientes que acudieron por emergencia lo hicieron luego de 24 horas de ocurrida la quemadura.

Contribuciones de autoria: GMWC, JEPR y CMS diseñaron el estudio; GMWC, ERH, JVCO y JRLM recolectaron la información del estudio; CMS, GMWC y JRLM analizaron e interpretaron la información; GMWC y CMS trabajaron en la redacción del artículo.

Conflictos de interés: Guillermo Martin Wiegering Cecchi, Enrique Rios Hidalgo, José Victor Córdova Orillo recibieron apoyo financiero de Sanofi Aventis de Perú para la recolección de datos del estudio; Juan Rafael Ludeña Muñoz recibió apoyo financiero de Sanofi Aventis para la recolección de datos de otro estudio de Sanofi Aventis de Perú. Cecilia Alexandra Medina Sanchez es Sanofi Perú Medical Head.

Fuentes de financiamiento: Investigación financiada por Sanofi Aventis de Perú

\section{REFERENCIAS BIBLIOGRÁFICAS}

1. Delgado J, Ramírez-Cardich ME, Gilman RH, Lavarello R, Dahodwala $\mathrm{N}$, Bazán A, et al. Risk factors for burns in children: crowding, poverty, and poor maternal education. Inj Prev. 2002;8(1):38-41.

2. Donroe J Gilman RH, Brugge D, Mwamburi M, Moore DA. Falls, poisonings, burns, and road traffic injuries in urban Peruvian children and adolescents: a community based study. Inj Prev. 2009;15(6):390-6. doi: 10.1136/ip.2008.019893. 
3. Plaza Heresi O. Tratamiento del paciente quemado hospitalario durante las primeras 48 horas: análisis de 36 casos en el Hospital Nacional Arzobispo Loayza. Horiz. méd. 2005;5(2):39-47.

4. Thomas-Gavelan E, Sáenz-Anduaga E, Ramos W, Sánchez-Saldaña L, Sialer Mdel C, Knowledge, attitudes and practices about sun exposure and photoprotection in outpatients attending dermatology clinics at four hospitals in Lima, Peru. An Bras Dermatol. 2011;86(6):1122-8.

5. Institute for Health Metrics and Evaluation. GBD 2018 Protocol: global burden of diseases, injuries, and risk factors. [Internet]. Seattle: IHME; 2018 [citado 13 febrero de 2019]. Disponible en: http://www. healthdata.org/gbd/about/protocol

6 Dirección General de Medicamentos, Insumos y Drogas, Ministerio de Salud. Evaluación de la situación de los medicamentos en el Perú [Internet]. Lima: DIGEMID, MINSA; 2006. Disponible en: http:// www.digemid.minsa.gob.pe/Upload/Uploaded/pdf/evasitmedicamentos.pdf

7. Papini R. Management of burn injuries of variousdepths.BMJ.2004;329(7458):15860. doi: 10.1136/bmj.329.7458.158.

8. Stylianou N, Buchan I, Dunn KW. A review of the international Burn Injury Database (iBID) for England and Wales: descriptive analysis of burn injuries 2003-2011. BMJ
Open. 2015;5(2):e006184. doi: 10.1136/ bmjopen-2014-006184.

9. Kakande I. Pattern and sensitivity of bacterial organisms infecting burns in $\mathrm{Mu}$ lago hospital, Kampala. East Afr Med J. 1978;55(5):223-7.

10. Cuttle L, Kempf M, Liu P-Y, Kravchuk O, Kimble RM. The optimal duration and delay of first aid treatment for deep partial thickness burn injuries. Burns. 2010;36(5):673-9. doi: 10.1016/j. burns.2009.08.002.

11. Schiefer JL, Perbix W, Grigutsch D, Zinser $\mathrm{M}$, Demir E, Fuchs PC, et al. Etiology, incidence and gender-specific patterns of severe burns in a German Burn Center - Insights of 25 years. Burns. 2016;42(3):687-96. doi: 10.1016/j.burns.2015.10.031.

12. Stewart BT, Lafta R, Esa Al Shatari SA, Cherewick M, Burnham G, Hagopian A, et al. Burns in Baghdad from 2003 to 2014: Results of a randomized household cluster survey. Burns. 2016;42(1):48-55. doi: 10.1016/j.burns.2015.10.002.

13. Morán Calvet MA. Características clinicoepidemiológicas de los accidentes por quemaduras en el municipio de Contramaestre. MEDISAN. 2010;14(3):311.

14. Atreya A, Nepal S, Kanchan T. Intentional burns - A form of gender based violence in Nepal. Burns. 2016;42(3):712.
15. Bhate-Deosthali P, Lingam L. Gendered pattern of burn injuries in India: a neglected health issue. Reprod Health Matters. 2016;24(47):96-103. doi: 10.1016/j. rhm.2016.05.004.

16. Aziz Z, Abu SF, Chong NJ. A systematic review of silver-containing dressings and topical silver agents (used with dressings) for burn wounds. Burns. 2012;38(3):307-18. doi: 10.1016/j.burns.2011.09.020.

17. Moncrief JA, Lindberg RB, Switzer WE, Pruitt BA. Use of topical antibacterial therapy in the treatment of the burn wound. Arch Surg. 1966;92(4):558-65.

18. Troche-Zaracho M, Maidana de Larrosa G, Lugo-Rodríguez G, Vera-Galván Z, Samaniego-Silva L. Utilización de antibióticos en el Centro Nacional del Quemado, Paraguay. Mem Inst Investig Cienc Salud. 2017;15(2):97-103.

19. Enoch S, Roshan A, Shah M. Emergency and early management of burns and scalds. BMJ.2009;338:b1037.

20. Castro RJA de, Leal PC, Sakata RK. Pain Management in Burn Patients. Braz J Anesthesiol Engl Ed. 2013;63(1):149-53. doi: 10.1016/S0034-7094(13)70206-X.

Correspondencia: Guillermo Martin Wiegering Cecchi.

Dirección: Los Ingenieros 872, Santiago de Surco; Lima, Perú

Teléfono: (511) 999263360

Correo electrónico:gwiegering@gmail.com

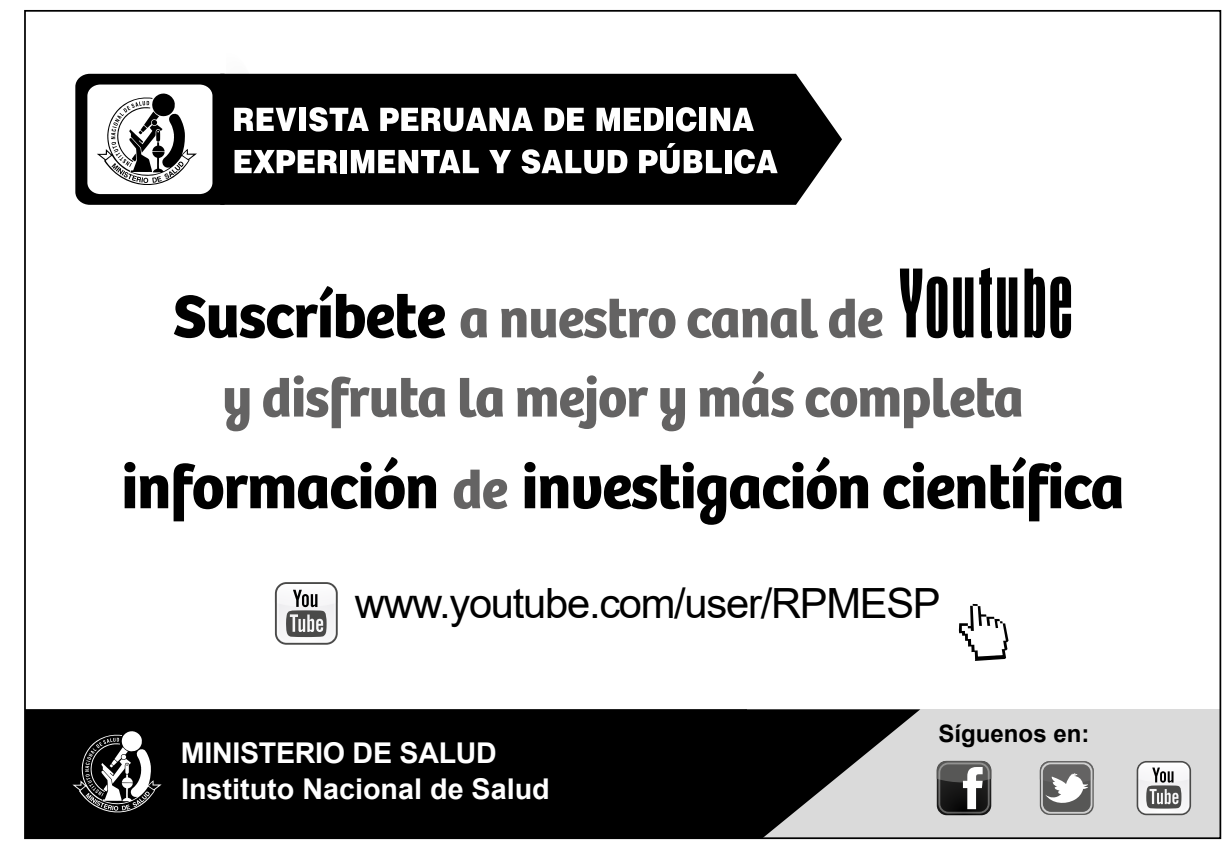

